\title{
CLEAN Technique in Strip-map SAR for High-Quality Imaging
}

\author{
Hirad Ghaemi ${ }^{3}$, Michele Galletti ${ }^{2}$, Thomas Boerner ${ }^{2}$, Frank Gekat ${ }^{1}$, Mats Viberg ${ }^{3}$ \\ ${ }^{1}$ SELEX Sistemi Integrati GmbH, Raiffeisenstrasse 10, D- 41470 Neuss, Germany \\ ${ }^{2}$ DLR, German Aerospace Center, Muenchner Strasse 20, 82234 Wessling, Germany \\ ${ }^{3}$ Chalmers University of Technology, Department of Signals and Systems, SE-41296, Gothenburg, Sweden \\ Tel: +49-8153-28-2368 \\ hirad@student.chalmers.se
}

\begin{abstract}
The maximum obtainable resolution of a stripmap synthetic aperture radar (SAR) system can be retained by simply avoiding weighting, or tapering, data samples in the along-track compression process. However, this will lead to hazardous artifacts caused by strong sidelobes of the corresponding adjacent scatterers whose interference might severely weaken the desired targets or even introduce false targets. On the other hand, some residual artifacts, even after tapering process, may still deteriorate the quality (contrast) of the SAR image. These issues can be remedied by applying the so-called CLEAN technique, which can mitigate these ill-effects in strip-map SAR imagery while maintaining the maximum resolution. This, indeed, is carried out as a post processing step, i.e., after the azimuth compression is accomplished, in the SAR system. The objective of this paper is to extend the CLEAN technique to strip-map SAR system to produce high-quality images with a very good along-track resolution. The algorithm is then applied to data from a ground-based circular SAR (CSAR) system to verify its implementation as well as this new application of the CLEAN technique. ${ }^{12}$
\end{abstract}

\section{TABLE OF CONTENTS}

1. INTRODUCTION

2. THE CLEAN AlgORITHM .....................................................2

3. AN EXPERIMENTAL EXAMPLE ...........................................4

4. CoNCLUSIONS …..........................................................6

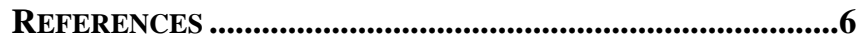

BIOGRAPHY .....................................................................................

\section{INTRODUCTION}

The long-aperture SAR systems capable of producing highresolution images, suffer from strong side lobe levels (SLL) introduced by the impulse response, or equivalently the corresponding processed pattern, of SAR as it occurs in long phased array imaging systems [1,5]. The interference of the strong sidelobe, associated with a group of adjacent scatterers, can result in destructive effects including breakup in the region of some strong scatterers and also creation of some new targets known as false targets with quite high amplitude levels [1]. Such unwanted effects, called artifacts, degrade the quality, or contrast, of the image severely. Tapering the samples within the synthetic aperture,

1

1978-1-4244-2622-5/09/\$25.00 @2009 IEEE.

${ }^{2}$ IEEEAC paper \#1000, Version 1, Updated September 10, 2008 performed by weighting function prior to along-track compression, is the simple and common solution to the current problem. However, there are a couple of drawbacks. The most important one is the deterioration of resolution which might be intolerable if the super resolution is desired. On the other hand, even though side-lobe levels (in particular the first one) are suppressed, the remaining artifacts can still have enough SNR, opposed to the weak scatterers, to affect the quality of the SAR image. Such circumstances mainly occur in long aperture, both synthetic and real one.

The CLEAN technique, first introduced for non-coherent radiation fields in radio astronomy [6], has been extended to long phased array imaging systems, which use coherent radiation fields, to tackle artifacts without any loss in resolution [1]. This latter technique, proposed for non-SAR imaging systems, is based on the assumption that the target consists of a set of isolated and point-like scatterers. Extended targets, i.e. contiguous ones such as dihedral scatterers, are not considered in the algorithm.

In this paper, we extend the coherent CLEAN to strip-map SAR imagery. The algorithm is developed in two dimensions and it covers largely distributed and contiguous scatterers in both range and along-track dimensions in addition to point-like scatterers. In other words, the CLEAN technique based on two-dimensional (2-D) feature extraction of distributed scatterers (as opposed to point scatterers), is going to be described. This is in fact the extended version of the two-dimensional CLEAN technique employed for feature extraction of point-like scatterers in inverse SAR (ISAR) autofocus approach reported in [4]. The mathematical procedures of non-linear least square (NLS) algorithm, presented for extracting features of both trihedral and dihedral reflectors, in one and two dimensions, are found to be of great use in the signal modeling step of the proposed algorithm [2]. However, those excessive iterations, associated with the NLS algorithm, are avoided.

The details of the proposed technique are explained in the following section. In the third section, an experimental example is demonstrated to guarantee the performance of this algorithm in eliminating artifacts without degrading the resolution. Finally, a few concluding remarks are made in the fourth section. 


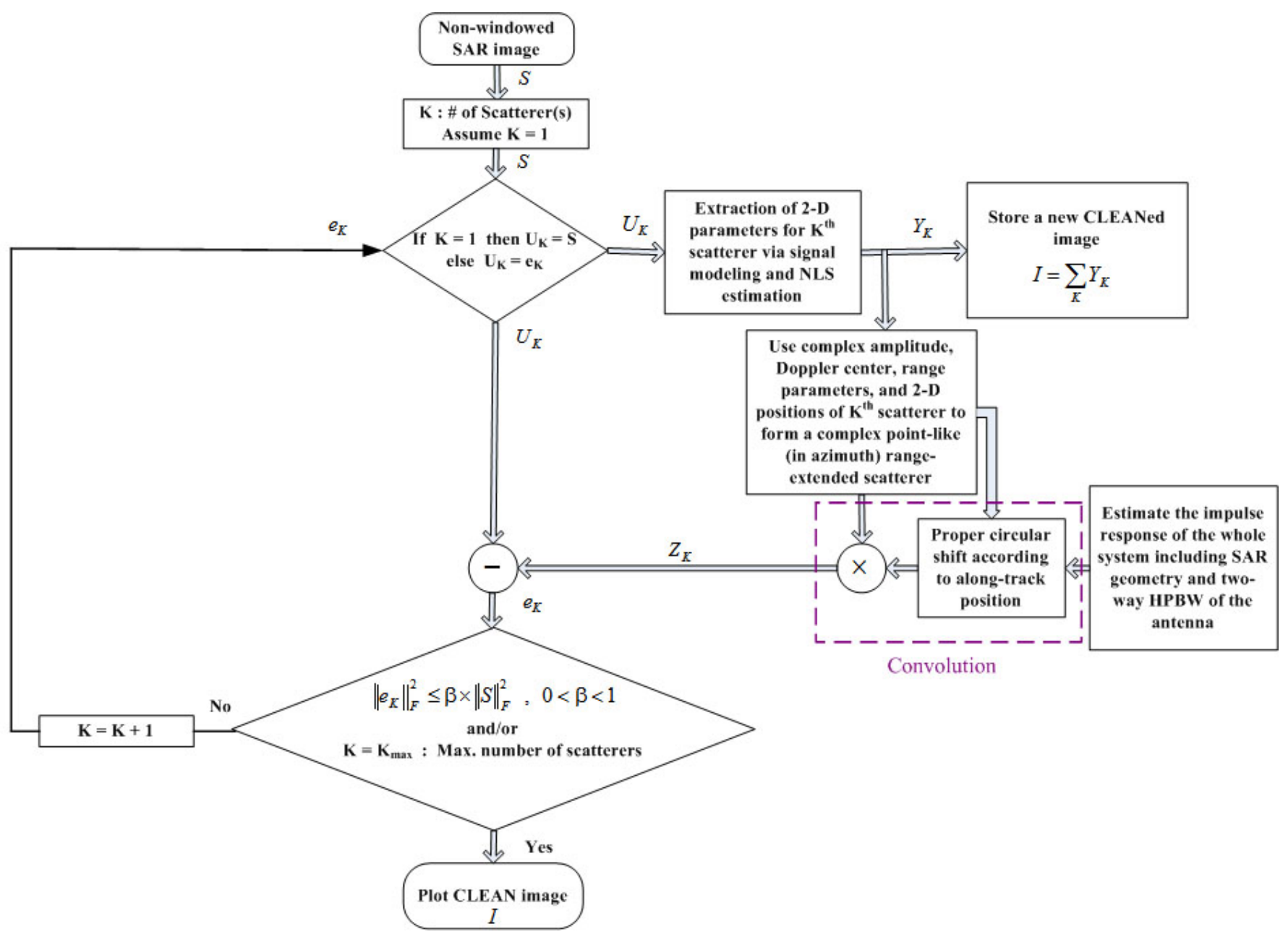

Figure 1 - The block diagram of the CLEAN algorithm for strip-map SAR imagery

\section{The Clean Algorithm}

The block diagram of the CLEAN algorithm for strip-map SAR is depicted in Fig.1. The process begins with the focused image, i.e. after the compression is performed in azimuth. Since the ultimate along-track resolution is desired, the aperture samples are not tapered (or weighted by window functions such as Hamming window prevalent in SAR processing) throughout the azimuth compression. The quality as well as dynamic range of the resultant image, therefore deteriorates around target areas due to artifacts arisen from side lobes. Similar to the CLEAN process in [1], the 2-D features of the brightest spot (or zone) on the focused image, need to be extracted. This is accomplished by the modified and extended version of NLS algorithm [2] (for solely one scatterer at a time), described in details later on. Here, it is assumed that the brightest spot is a real target and not an artifact, otherwise the process will fail (this, indeed, is the basic assumption in the CLEAN algorithm [1]). The complex samples of the extracted scatterer are stored in a different variable. The impulse response (the amplitude pattern) of the whole SAR system including SAR geometry and two-way half-power beamwidth (HPBW) of the antenna is to be determined to estimate the pattern of sidelobes imposed by the radar system as well as by the SAR processor. The 3-dB mainlobe can be considered to be of Gaussian form with a certain angular resolution dictated by the antenna's parameters. In other words, the impulse response of the SAR system is an image produced by an ideal point source (i.e. stationary, coherent, and strong point reflector with unit amplitude) [3]. Afterwards, the amplitude pattern must be shifted to the correct along-track position, already estimated by 2-D NLS and then multiplied by the complex samples of the strongest target expanded in range but regarded as a point-like scatterer in azimuth. In general, the system impulse response must be convolved with the target reflectivity function, instead of performing shift and multiplication (encircled by dashed lines in Fig.1), in order to obtain the final along-track pattern (including both mainlobe and sidelobes) of the corresponding target in the image domain [3]. However, for the sake of simplicity, the target is treated as a coherent point-like scatterer in azimuth, irrespective of its range extension, which implicitly means that the resolution as well as the overall impulse response is 
manipulated and, therefore, determined by the SAR system. Now, the 2-D complex samples of the estimated pattern associated with the brightest spot (or small zone), must be subtracted from the complex image. In contrast to [1], herein, the subtraction, or CLEANing, is performed in image domain rather than in the aperture domain (or equivalently in the field domain). After subtraction, the new image is devoid of the strongest scatterer and its sidelobes. By following the previously stated steps, the next brightest point is similarly searched for and processed. The iterative CLEAN can continue down to the noise floor. However, it is highly likely that the CLEAN process fails to function properly, e.g. it might introduce false targets to the new image, due to low signal to noise ratio (SNR) of weak sources whose respective impulse responses are affected severely by the available noise in the radar system. In this paper, the iterative process is simply terminated when the residual energy, i.e. the energy of the image left after several subtractions, violates a preset energy threshold (a certain percentage of total energy in the primary image) and/or the number of iterations fulfils a desired number of scatterers. Alternatively, one may set the thresholds, relative and absolute ones, developed in [1] based on both SNR and the variance of the phase error introduced by the imaging system, propagation anomalies, multipath, etc.

In addition to the SNR, the coherency of the target during the SAR illumination, or integration interval, can limit the performance of the CLEAN algorithm. It is also worth to note that the phase error in SAR must be estimated and properly included in determination of the impulse response of the whole system (see [3]) before it is proceeded with the convolution and subtraction. Since the phase error (multiplicative noise) controls the properties associated with mainlobe and sidelobes (their positions, their widths, and their relative amplitude levels) [7], its variance, if high, can make the CLEAN technique appear totally ineffective unless it is either mitigated or estimated and involved in the CLEAN process. The corresponding step related to phase error, however, is not included in Fig.1, because it is assumed to be negligible.

In the following, 2-D NLS algorithm is formulated in general form, similar to [2]. However, this algorithm is intended to be used for feature extraction of a single scatterer at each iteration of CLEAN.

The measured samples $y(m, n)$ in along-track and range dimension (denoted by variables $m$ and $n$, respectively) can be expressed as

$$
\begin{gathered}
y(m, n)=s(m, n)+v(m, n) \\
m=0,1, \cdots, M-1 \quad, \quad n=0,1, \cdots, N-1
\end{gathered}
$$

where $v(m, n)$ are the 2-D complex samples of noise and $s(m, n)$ are the model-based samples given as

$$
s(m, n)=\sum_{k=1}^{K} \alpha_{k} g_{k}(m) h_{k}(n)
$$

In the above equation, $g$ and $h$ for the $k$ th scatterer are

$$
\begin{aligned}
& g_{k}(m)=\exp \left(j 2 \pi P R I \cdot f_{a_{k}} m\right) \\
& \times \exp \left(-\frac{\pi^{2}}{4 \ln 2}\left[P R I \cdot \Delta_{a_{k}} \cdot\left(m-d_{a_{k}}\right)\right]^{2}\right) \\
& h_{k}(n)=\exp \left(j 2 \pi R S I \cdot f_{r_{k}} n\right) \\
& \quad \times \exp \left(-\frac{\pi^{2}}{4 \ln 2}\left[R S I \cdot \Delta_{r_{k}} \cdot\left(n-d_{r_{k}}\right)\right]^{2}\right)
\end{aligned}
$$

$P R I$ and $R S I$ stand for the pulse repetition interval (sampling interval in cross range) and the range sampling interval, respectively, and $k$ is defined as the number of scatterers. Moreover, $\alpha_{k},\left\{f_{a_{k}}, f_{r_{k}}\right\},\left\{\Delta_{a_{k}}, \Delta_{r_{k}}\right\}$, and $\left\{d_{a_{k}}, d_{r_{k}}\right\}$ are the complex amplitude, 2-D frequency center , 2-D spectrum width, and 2-D delay of the $k$ th scatterer respectively. Subscripts " $a$ " and " $r$ " are representing azimuth and range, respectively.

We use bold letters $\mathbf{y}$ and $\mathbf{G}_{k}$ to represent $M \times N$ matrices. The Non-Linear Least-Squares (NLLS) estimate of the target features are obtained by minimizing the following cost function

$$
\begin{gathered}
C_{1}\left(\left\{\alpha_{k}, f_{a_{k}}, f_{r_{k}}, \Delta_{a_{k}}, \Delta_{r_{k}}, d_{a_{k}}, d_{r_{k}}\right\}_{k=1}^{K}\right) \\
=\left\|\mathbf{y}-\sum_{k=1}^{K} \mathbf{G}_{k} \alpha_{k}\right\|_{F}^{2}
\end{gathered}
$$

in which " \|\|$_{F}$ "denotes Frobenius norm and

$$
\begin{gathered}
\mathbf{G}_{k}=\mathbf{g}_{k} \times \mathbf{h}_{k}^{T} \\
\mathbf{g}_{k}=\left[\begin{array}{llll}
g_{k}(0) & g_{k}(1) & \cdots & g_{k}(M-1)
\end{array}\right]^{T} \\
\mathbf{h}_{k}=\left[\begin{array}{llll}
h_{k}(0) & h_{k}(1) & \cdots & h_{k}(N-1)
\end{array}\right]^{T}
\end{gathered}
$$

The cost function $C_{1}$ is a non-linear function of all parameters except $\alpha_{k}$. Thus, rather than trying to solve for all parameters simultaneously, an iterative approach is used, where the parameters of one scatterer are considered at a time. Assume the parameters of all scatterers but the $k$ th are known (or previously estimated). Minimizing $C_{1}$ with respect to $\alpha_{k}$ then yields its estimate $\hat{\alpha}_{k}$ which is 


$$
\widehat{\alpha}_{k}=\frac{\mathbf{g}_{k}^{H} \mathbf{y}_{k} \overline{\mathbf{h}}_{k}}{\left\|\mathbf{g}_{k}\right\|^{2}\left\|\mathbf{h}_{k}\right\|^{2}}
$$

where

$$
\mathbf{y}_{k}=\mathbf{y}-\sum_{i=1, i \neq k}^{K} \alpha_{i} \mathbf{G}_{i}
$$

" $H$ " denotes the Hermitian matrix operation and the overbar represents complex conjugate. Besides, "|l||" denotes Euclidean norm.

By inserting (9) into (5) and performing some simplification, a new cost function $C_{2}$ is to be maximized over the rest of unknown parameters of $k$ th scatterer in order to realize the minimization of $C_{1}$ over the same parameters, i.e.

$$
C_{2}\left(f_{a_{k}}, f_{r_{k}}, \Delta_{a_{k}}, \Delta_{r_{k}}, d_{a_{k}}, d_{r_{k}}\right)=\frac{\left\|\mathbf{g}_{k}^{H} \mathbf{y}_{k} \overline{\mathbf{h}}_{k}\right\|^{2}}{\left\|\mathbf{g}_{k}\right\|^{2}\left\|\mathbf{h}_{k}\right\|^{2}}
$$

Since at each iteration of CLEAN process only one dominant scatterer is searched for, $K$ is equal to one and $\boldsymbol{y}_{k}$ is replaced by $\boldsymbol{y}$. To maximize (11) in general, a sixdimensional search over the parameter space is required. To accomplish this, we follow the alternating procedure [2] in which one parameter estimate is updated while the rest are considered fixed. Note that the 2-D frequency center $\left\{f_{a}, f_{r}\right\}$, can be readily estimated by finding the peak location of the 2-D fast Fourier transform (FFT) of the 2-D function $\boldsymbol{P}$, when the other parameters remain constant.

$$
\boldsymbol{P}=\left(\mathbf{g}_{0} \cdot[\mathbf{1}]_{N}^{T}\right) \otimes \mathbf{y} \otimes\left([1]_{M} \cdot \mathbf{h}_{0}^{T}\right)
$$

$\mathbf{g}_{0}$ and $\mathbf{h}_{0}$ are the vectors $\mathbf{g}$ and $\mathbf{h}$ calculated at center frequencies $f_{\mathrm{a}}$ and $f_{\mathrm{r}}$ set to zero, respectively. In (12), " $[\mathbf{1}]_{L}$ " is defined as a column-wise unit vector with length $L$ and “ $\otimes$ " denotes element-by-element matrix multiplication. Here zero padding prior to the 2-D FFT is necessary for higher accuracy.

To accelerate the estimation process, which is quite tedious and time-consuming, the spectral widths $\left\{\Delta_{a}, \Delta_{r}\right\}$ are roughly determined via 3-dB bandwidth of the abovementioned function $\boldsymbol{P}$ over the 2-D frequency spectrum as it is done by the fast Fourier transform based (FFTB) algorithm outlined in [2]. Eventually, the delays $d_{\mathrm{a}}$ and $d_{\mathrm{r}}$ are estimated by golden section search algorithm (using the corresponding MATLAB function) and a simple timedomain search (not interpolated, therefore its accuracy is limited by sampling interval), respectively, to maximize $C_{2}$. Throughout the search process, a few iterations might turn out to be necessary to gain a better estimation of the parameters.

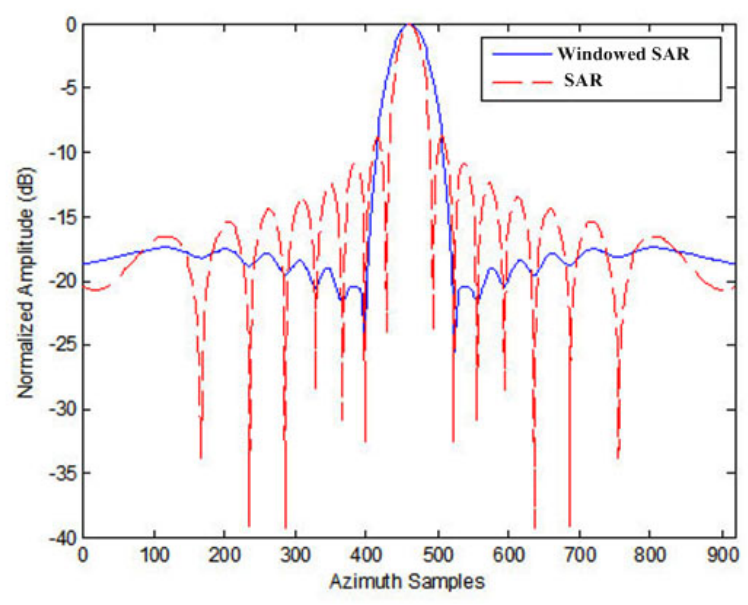

Figure 2 - The impulse response of the SAR system with and without tapering

\section{AN EXPERIMENTAL EXAMPLE}

The experimental data, exploited in this section, have been collected by SELEX-SI Gematronik Inc. using a groundbased rotating Doppler weather radar with a small antenna [8]. The corresponding antenna has a wide horizontal beamwidth and it has been installed on a fairly large platform and therefore, it has been capable of producing a quite long synthetic aperture with an integration time around $700 \mathrm{~ms}$.

One of the objectives of this setup is to take advantage of the circular path of the sensor, which can be thought of as a strip-map CSAR, to produce high resolution and clear images of the far-distance ground clutter affecting desired targets $^{3}$.

As plotted in Fig.2, the azimuth impulse response of the CSAR system (without applying any weighting function, depicted by the dashed line) has quite high SLLs. Although they are suppressed by applying a Hamming window to the samples prior to coherent integration, the tapered impulse response (plotted by the solid line in Fig.2) still suffers from remaining side-lobes, not to mention the fact that the resolution has deteriorated.

\footnotetext{
4

${ }^{3}$ Based on a collaborative project between SELEX-SI and DLR
} 


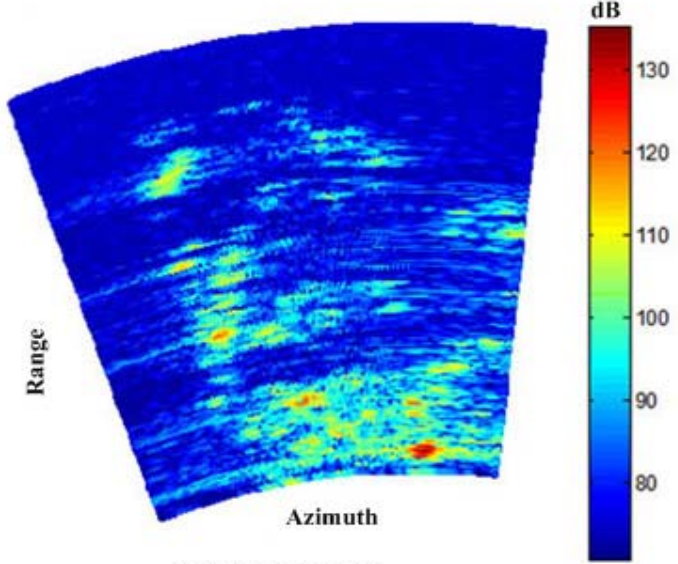

(a) SAR image

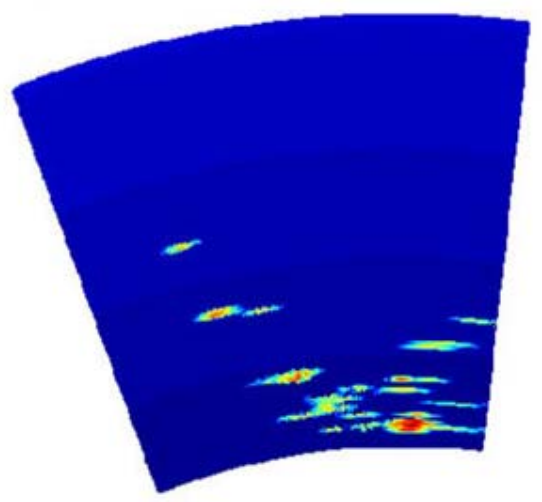

(c) CLEANed SAR image for $\beta=0.2$

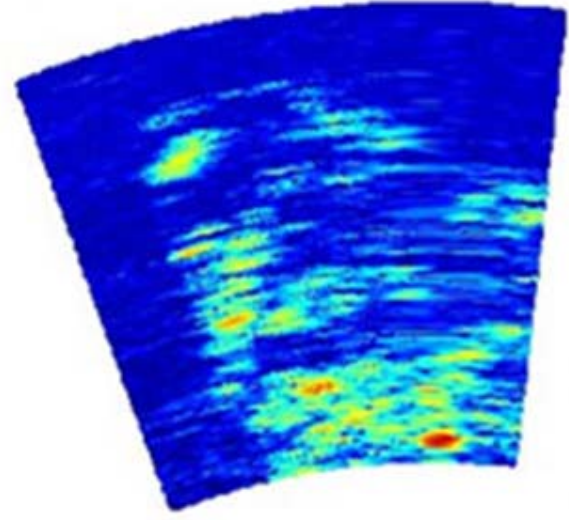

(b) Windowed SAR image

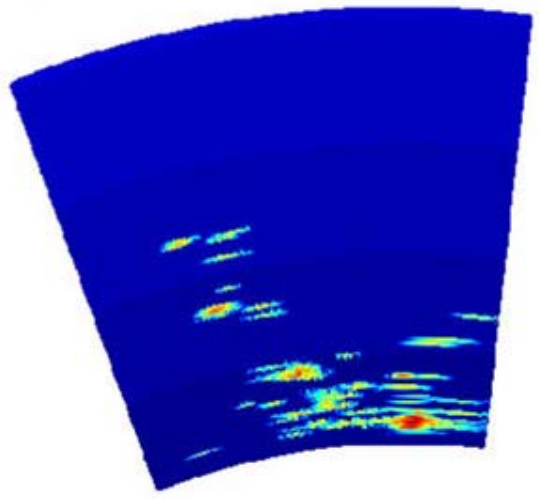

(d) CLEANed SAR image

for $\beta=0.15$

Figure 3 - The SAR, windowed SAR, and CLEANed SAR images

Fig.3 (a) and (b) illustrate SAR images, containing strong clutter on the ground, obtained by using FFT and windowed FFT, respectively. It is easy to notice that the quality of the SAR image, even after windowing, is still poor due to strong artifacts.

The improved images using the CLEAN technique outlined in Fig.1 with $\beta$ (a portion of the total signal energy available in the primary image) equal to 0.2 and 0.15 , are shown in Fig.3 (c) and (d), respectively. It is apparent that the contrast as well as dynamic range around the target areas is increased considerably. In other words, the several adjacent targets can now be better identified and distinguished from one another. In addition, the resolution remains intact, as anticipated. For clarification, a few alongtrack samples of both SAR and CLEANed SAR, at some specific range bins, are depicted in Fig.4. In one of the plots, the constructive interference of sidelobes associated with a couple of strong adjacent scatterers, has created false targets and also it has caused a break-up in one of the targets. These strong artifacts, as an example, are encircled with a dashed line in Fig.4.
Since the current paper basically aims at the extension of the CLEAN algorithm and its new implementation, here, also for the sake of simplicity, a constant threshold is defined based on the residual energy left out of the CLEAN process rather than moving-level thresholds investigated in [1]. Indeed, the deeper the CLEAN process proceeds, the more targets are detected with lower SNR and a higher probability of false target detection as well. In an attempt to find out the thresholds in [1], the dominant phase error will be the aperture-dependent phase error, the platform motion perturbation and the error introduced by propagation effects. The variance of this phase error and the SNR of the detected target affect the threshold level at each iteration of the CLEAN algorithm. 

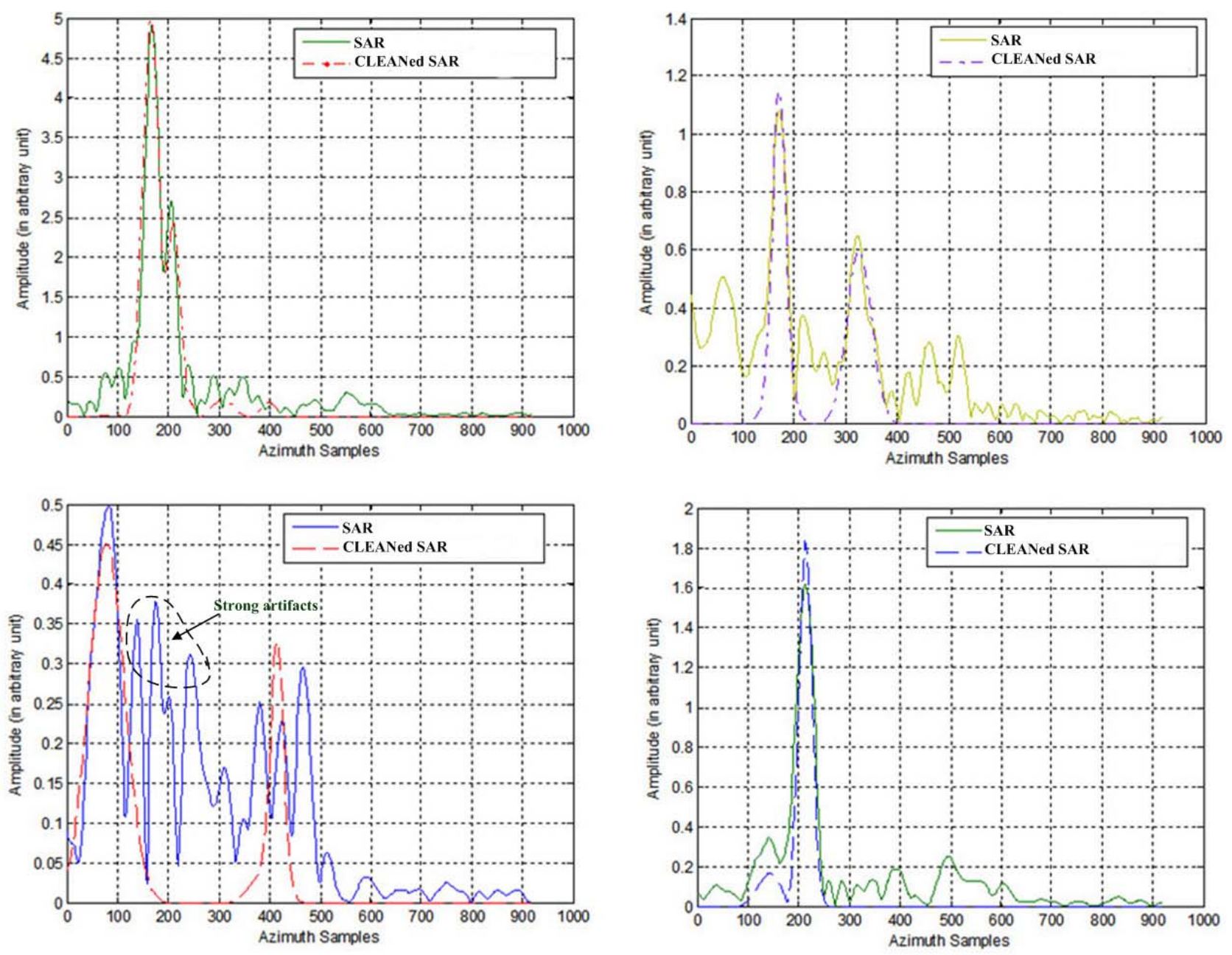

Figure 4 - Along-track samples of SAR and CLEANed SAR images at some certain range bins

\section{CONCLUSIONS}

In this paper, the new and simple extension of the CLEAN technique to strip-map SAR has been studied. The algorithm has been proposed in two dimensions. A modified 2-D parameter estimation has been developed via NLS algorithm to determine the important parameters involved in the CLEAN process. The impulse response of the SAR system specifies the azimuth pattern consisting of main lobe and side lobes, as long as the SNR is high enough and the system is devoid of serious phase errors.

An experimental example has been demonstrated to verify that the image quality and dynamic range has been improved by this extended CLEAN algorithm, while the maximum resolution has been preserved. Besides, the new application to strip-map CSAR has been investigated.

\section{REFERENCES}

[1] J. Tsao, and B. D. Steinberg, "Reduction of sidelobe and speckle artifacts in microwave imaging: the CLEAN technique," IEEE Trans. Antennas Prop., vol.36, pp.543-556, April 1988.

[2] Z. S. Liu and J. Li, "Feature extraction of SAR targets consisting of trihedral and dihedral corner reflectors," IEE Proc. Radar, Sonar Navig., vol. 145, pp. 161-172, June 1998.

[3] W. M. Brown, "Synthetic aperture radar," IEEE Trans. Aerosp. Electron. Sys., vol.3 ,pp. 217-229, March 1967.

[4] J. Li, R. Wu., and V. C. Chen, "Robust autofocus algorithm for ISAR imaging of moving targets," IEEE Trans. Aerosp. Electron. Sys., vol. 37,pp. 1056-1068, July 2001. 
[5] B. D. Steinberg, Microwave imaging with large antenna arrays. New York: Wiley,1983.

[6] J. A. Högbom, "Aperture synthesis with a non-regular distribution of interferometer baselines,"Astron Astrophys Suppl.,vol.15, pp.417-426,.1974.

[7] C. A. Greene and R. T. Moller, "The effect of normally distributed random phase errors on synthetic array gain patterns," IRE Trans. Military Electronics., pp. 130-139, April 1962

[8] H. Ghaemi, M. Galletti , T. Boerner, F. Gekat, and M. Viberg, "Synthetic aperture weather radar," M.Sc. Thesis, DLR (German Aerospace Center), Wessling, July 2008.

\section{BIOGRAPHY}

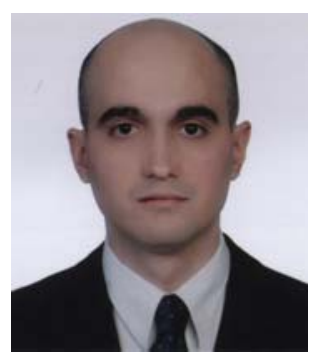

Hirad Ghaemi received the B.Sc. and M.Sc. degree in electrical engineering from Iran University of Science and Technology in 2003 and 2005, respectively, and M.Sc. in digital communication systems and technology from Chalmers University of Technology, Sweden, in 2008. He gained some work experiences in active microwave circuit design, radar system engineering, microwave and antenna measurement and signal processing in SAR. His recent project carried out in DLR (German Aerospace Center), was on SAR signal processing in polarimetric Doppler weather radars. His research interests include signal processing in SAR imagery, radar system design and simulation, and active microwave circuit/MMIC design.

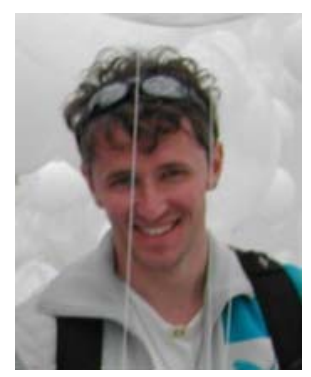

Michele Galletti received the Laurea degree in physics (110/110 e lode) from the Alma Mater Studiorum Università di Bologna, Bologna, Italy in 2003. In 2004 and 2005 he was a young scientist within the EU-funded project AMPER, working on applications of radar polarimetry. Since 2006 he has been with the Microwaves and Radar Institute of the German Aerospace Center (DLR-HR-RK). $H$ is research interests are radar polarimetry and radar system design. He is currently enrolled in a Ph.D program in electrical engineering.

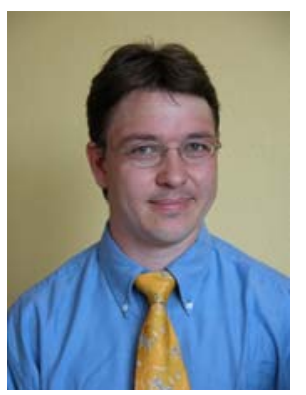

Thomas Boerner received the diploma degree in physics from the University of Munich, Germany in 1996, followed by a Ph.D. in Geography (remote sensing) in
2000. Since 2005 he is the head of the propagation and scattering group at the Microwaves and Radar Institute of the German Aerospace Center (DLR-HR-RK). His current activities are the management and scientific supervision of quality assessment of SAR data, studies about atmospheric propagation effects in weather radar and SAR data, and investigations in new radar sensor concepts and designs.

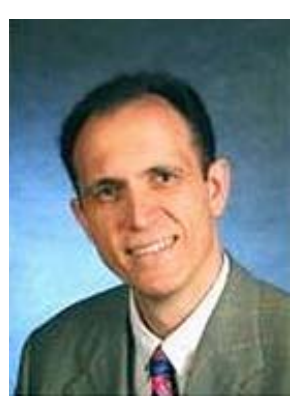

Frank Gekat is Director of Development at Selex Sistemi Integrati, Neuss. He has directed the development of several meteorological radar systems including the METEOR 500/1500 and 600/1600 series. He previously served as Business Development Manager for Particle Accelerator Systems at Siemens AG, Germany and as Development Engineer at the Institute of Technical Physics of the DLR, Germany. He earned a Dipl.-Ing. and a Dr.-Ing. Degree from the Ruhr-Universität Bochum, Germany.

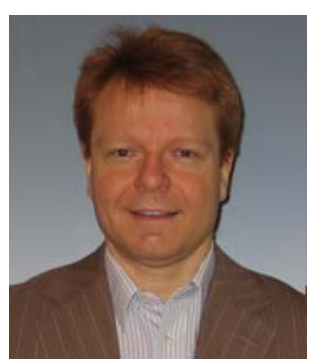

Mats Viberg received the $P h D$ degree in Automatic Control from Linköping University, Sweden in 1989. During 1989-1992 he was an Assistant Professor in Linköping University and 1992-1993 he held visiting Scholarships at the Information Systems Laboratory, Stanford University, and the Department of ECE, Brigham Young University, USA. Since 1993, Dr. Viberg is a professor of Signal Processing at the Department of Signals and Systems (formerly Applied Electronics), Chalmers University of Technology, Sweden. During 1999-2004 he served as the Chair of the same department. Dr. Viberg's research interests are in Statistical Signal Processing and its various applications, including Antenna Array Signal Processing, System Identification, Wireless Communications, Radar Systems and Automotive Signal Processing. Dr. Viberg was the Chair of the IEEE SP Society's Technical Committee on Signal Processing Theory and Methods 2001-2003. He served as an Associate Editor of the IEEE Transactions on Signal Processing 2004-2005, in the IEEE SP Awards board 2005-2007, and since 2006 in the IEEE SP Technical Committee on Sensor Array and Multichannel. He was the Technical Chair of the IEEE Workshop on Statistical Signal Processing (SSP) 2005, and Technical Co-Chair of the IEEE Workshop on Computational Advances in Multisensor Adaptive Processing (CAMSAP) 2007. Dr. Viberg has received 2 Paper Awards from the IEEE Signal Processing Society (1993 and 1999 respectively), and the Excellent Research Award from the Swedish Research Council (VR) in 2002. Dr Viberg is a Fellow of the IEEE since 2003, and his research group received the 2007 EURASIP European Group Technical Achievement Award. In 2008, Dr. Viberg 
was elected into the Royal Swedish Academy of Sciences (KVA). 